\title{
Protective effects of human umbilical cord blood-derived mesenchymal stem cells against dexamethasone-induced apoptotic cell death in hair follicles
}

\author{
DONG HO BAK ${ }^{1 *}$, ESTHER LEE ${ }^{2,3 *}$, MI JI CHOI ${ }^{2,3}$, BYUNG CHUL LEE $^{2,3}$, TAE-RIN KWON $^{2,3}$, \\ JONG-HWAN KIM ${ }^{2,3}$, EUN SU JEON ${ }^{4}$, WONIL OH ${ }^{4}$, SEOG KYUN MUN ${ }^{5}$, BYUNG CHEOL PARK ${ }^{6}$, \\ JUNGTAE NA ${ }^{2,3}$ and BEOM JOON KIM ${ }^{2,3}$
}

\begin{abstract}
${ }^{1}$ Advanced Radiation Technology Institute, Korea Atomic Energy Research Institute, Jeongeup, North Jeolla 56212; ${ }^{2}$ Department of Dermatology, College of Medicine; ${ }^{3}$ Department of Medicine, Graduate School, Chung-Ang University, Seoul 06973; ${ }^{4}$ Biomedical Research Institute, R\&D Center, MEDIPOST Co., Ltd., Seongnam, Gyeonggi 13494; ${ }^{5}$ Department of Otorhinolaryngology, College of Medicine, Chung-Ang University, Seoul 06973; ${ }^{6}$ Department of Dermatology, Dankook Medical College, Cheonan, South Chungcheong 31116, Republic of Korea
\end{abstract}

Received July 23, 2019; Accepted November 29, 2019

DOI: $10.3892 /$ ijmm.2019.4447

\begin{abstract}
Alopecia is a common and distressing condition, and developing new therapeutic agents to prevent hair loss is important. Human umbilical cord blood-derived mesenchymal stem cells (hUCB-MSCs) have been studied intensively in regenerative medicine. However, the therapeutic potential of these cells against hair loss and hair organ damage remains unclear, and the effects of hUCB-MSC transplantation on hair loss require evaluation. The current study aimed to investigate the effects of hUCB-MSCs on hair regression in vivo and restoration of anagen conduction on hair growth in vitro. The effects of hUCB-MSCs were explored in mouse catagen induction models using a topical treatment of $0.1 \%$ dexamethasone to induce hair regression. Dexamethasone was also used to simulate a stress environment in vitro. The results demonstrated that
\end{abstract}

Correspondence to: Professor Jungtae $\mathrm{Na}$ or Professor Beom Joon Kim, Department of Dermatology, College of Medicine, 102 Heukseok-ro, Dongjak, Seoul 06973, Republic of Korea

E-mail: pugokjebi@gmail.com

E-mail: beomjoon74@gmail.com

${ }^{*}$ Contributed equally

Abbreviations: hDPCs, human dermal papilla cells; hUCB-MSCs, human umbilical cord blood-derived mesenchymal stem cells; GC, glucocorticoid; HPA, hypothalamic-pituitary-adrenal; GR, glucocorticoid receptor; H\&E, hematoxylin and eosin; HCS, hair cycle score; VEGF, vascular endothelial growth factor; IHC, immunohistochemistry; IF, immunofluorescence; MMP, mitochondrial membrane potential; PARP, poly (ADP-ribose) polymerase; UPR, unfolded protein response

Key words: hair follicle, hUCB-MSCs, alopecia, stem-cell therapy, glucocorticoid
hUCB-MSCs significantly prevented hair regression induced by dexamethasone topical stimulation in vivo. Additionally, hUCB-MSCs significantly increased the proliferation of human dermal papilla cells (hDPCs) and HaCaT cells, which are key constituent cells of the hair follicle. Stimulation of vascular endothelial growth factor secretion and decreased expression of DKK-1 by hUCB-MSCs were also observed in hDPCs. Restoration of cell viability by hUCB-MSCs suggested that these cells exerted a protective effect on glucocorticoid stress-associated hair loss. In addition, anti-apoptotic effects and regulation of the autophagic flux recovery were observed in $\mathrm{HaCaT}$ cells. The results of the present study indicated that hUCB-MSCs may have the capacity to protect hair follicular dermal papilla cells and keratinocytes, thus preventing hair loss. Additionally, the protective effects of hUCB-MSCs may be resistant to dysregulation of autophagy under harmful stress.

\section{Introduction}

The human umbilical cord is often considered to be a substitute source of stem or progenitor cells (1). Acquisition of Human umbilical cord blood-derived mesenchymal stem cells (hUCB-MSCs) is more efficient compared with bone marrow-derived MSCs as these cells are acquired by non-invasive methods (2). Transplantation of hUCB-MSCs is a well-established alternative treatment for a wide spectrum of diseases, including visual impairment, glioma, ischemic brain damage, liver disease and cartilage degeneration (3-7).

Alopecia is the hereditary thinning of hair induced by androgens in men and women (8). In alopecia, the size of the hair follicles is reduced, and the hair shaft thins and ultimately disappears (9). The anagen or growth phase gradually shortens, revealing smaller follicular tissue and irregularly shaped hair shafts covering the scalp (10). Based on morphological changes observed during the degeneration of the hair follicle, the regression of this mini-organ is predominantly caused by coordinated keratinocyte apoptosis (9). 
Glucocorticoid (GC) levels are regulated by the adrenocorticotropic hormone, which is regulated by the hypothalamic-pituitary-adrenal (HPA) axis (11). A previous study has suggested that in keratinocytes, the glucocorticoid receptor (GR) is associated with the expression of a variety of genes, including those involved in apoptosis, cell adhesion, lipid metabolism and formation of the stratum corneum (12). The skin and hair are targets of HPA axis activity and also produce GCs through their own neuroendocrine systems; this nominal 'peripheral' HPA axis is associated with hair loss $(13,14)$. Previous studies demonstrated that corticotropin-releasing hormone significantly inhibited hair shaft elongation in vitro, induced hair regression, inhibited hair matrix keratinocyte proliferation and upregulated apoptosis of root sheath cells ex vivo $(15,16)$.

The present study aimed to demonstrate the preventive effects exerted by hUCB-MSCs on hair loss and the mechanisms that underlie alopecia prevention by investigating the effect of hUCB-MSCs on dexamethasone (Dex)-induced hair loss in mouse catagen induction models. The effects of hUCB-MSCs on human dermal papilla cells (hDPCs) and $\mathrm{HaCaT}$ cells under Dex-induced stress were also investigated to elucidate the molecular mechanisms underlying hair follicle protection.

\section{Materials and methods}

Animals. All animal procedures were approved by the Institutional Animal Care and Use Committee (IACUC) of Chung-Ang University (2018-03-20) and performed in accordance with the Guide for the Care and Use of Laboratory Animals published by the US National Institutes of Health (17). Female C57/BL6 mice (6 weeks old) were obtained from Saeron Bio, Inc. and allowed to adapt for one week with ad libitum feeding. The animals were randomly divided into 3 groups (n=6) as follows: i) Normal control group (NoC), saline injection; ii) $0.1 \%$ Dex only treatment group (Dex), $0.1 \%$ Dex + saline injection; and iii) hUCB-MSC treatment group (Dex + hUCB-MSCs), 0.1\% Dex + hUCB-MSCs ( 8 sites/head, $100 \mu \mathrm{l} /$ site). The mice were anesthetized with Zoletil $50(50 \mathrm{mg} / \mathrm{kg})$ and xylazine $(10 \mathrm{mg} / \mathrm{kg})$ by IP injection as described previously $(18,19)$. Hair cycle synchronization of mouse models was induced by taping of the dorsal skin during the telogen phase of the hair cycle, as described previously (20). On the final day of depilation, the percentage of dorsal skin color change on the back of each mouse was evaluated by comparing the change in skin color progression, and a hair cycle score (HCS) was calculated using the formula described by Müller-Röver et al (21).

Histological analysis. Histology was analyzed by hematoxylin and eosin (H\&E) staining. Immunohistochemistry (IHC) and immunofluorescence (IF) were performed on $4 \%$ paraformaldehyde-fixed and paraffin-embedded sections. The tissues were blocked 3\% BSA and 5\% normal goat in Tris-buffered saline with $0.1 \%$ Tween 20 (TBS-T, pH 7.4) for $2 \mathrm{~h}$ at room temperature. For IHC, antibodies against $\beta$-catenin (cat. no. 610154; 1:500; BD Biosciences) and Dickkopf WNT signaling pathway inhibitor 1 (DKK-1; cat. no. ab109416; 1:500; Abcam) were used. The sections were washed with Tris-buffered saline containing $0.1 \%$ Tween 20 for 10 min three times, and the primary antibodies were detected with biotinylated Goat Anti-Mouse IgG Antibody (cat. no. BP-9200; 1:1,000; Vector Laboratories) and biotinylated Goat Anti-Rabbit IgG Antibody (cat. no. BP-9500; 1:1,000; Vector Laboratories) for $4 \mathrm{~h}$ at room temperature plus a streptavidin-peroxidase complex (Vector Laboratories, Inc.) and brown FAST DAB (Thermo Fisher Scientific, Inc.) staining. Slides were observed by light microscopy (DM750; Leica Microsystems GmbH) in five consecutive fields at x100 magnification. Antibodies against microtubule-associated protein 1 light chain $3 \beta$ (LC3BI/II; cat. no. PM036; 1:500; MBL International Co.) and p62 (cat. no. PM045; 1:500, MBL International Co.) were used for IF. Samples were mounted on slides, and images were acquired using a confocal microscope (LSM700; Zeiss AG) in five consecutive fields at magnification, $\mathrm{x} 200$.

Preparation of $h U C B-M S C s$. This study was approved by the Institutional Review Board of MEDIPOST Co., Ltd. Collection of hUCB and isolation and culture of hUCB-MSCs were performed as previously described (22). Mononuclear cells were isolated from hUCBs by centrifugation on a Ficoll-Hypaque gradient (density, $1.077 \mathrm{~g} / \mathrm{cm}^{3}$; Sigma-Aldrich; Merck KGaA). Cells were then seeded in culture flasks at $5 \times 10^{5}$ cells $/ \mathrm{cm}^{2}$. Following the formation of spindle-shaped cell colonies, cells were reseeded for expansion. hUCB-MSCs were cultured in $\alpha$ MEM medium (Gibco; Thermo Fisher Scientific, Inc.) supplemented with 10\% FBS (Gibco; Thermo Fisher Scientific, Inc.) and gentamycin (Gibco; Thermo Fisher Scientific, Inc.) at $37^{\circ} \mathrm{C}$ in a humidified atmosphere containing $5 \% \mathrm{CO}_{2}$ and $3 \% \mathrm{O}_{2}$. Cells were passaged when they reached $80 \%$ confluency and used either for experiments or redistribution to new culture plates. In all experiments, hUCB-MSCs were used at passage 6 .

Measurement of apoptosis by TUNEL assay. A TUNEL assay was performed on mouse dorsal skin tissue using the DeadEnd fluorometric in situ cell death detection kit (Promega Corporation) according to the manufacturer's protocol in order to analyze DNA fragmentation, which is indicative of apoptosis. DAPI was used to visualize the nuclei. Images were acquired using a confocal microscope (LSM700; Zeiss AG).

Cell viability assay. $\mathrm{hDPCs}$ were obtained from CEFO Co., Ltd. (cat. no. CB-HDP-001) and cultured for 6 passages prior to use in experiments. The experiments were conducted using short incubation periods as long in vitro culture resulted in hDPCs losing their original characteristics and functions. HaCaT cells, which are immortalized human keratinocytes, were obtained from Addexbio Technologies (cat. no. T0020001). The two cell lines were cultured in Dulbecco's Modified Eagle's Medium (DMEM; Welgene, Inc.) supplemented with 10\% FBS (Gibco; Thermo Fisher Scientific, Inc.) and 1\% Penicillin-Streptomycin (Gibco; Thermo Fisher Scientific, Inc.). During the culture, $5 \% \mathrm{CO}_{2}$ was continuously supplied while maintaining the temperature at $37^{\circ} \mathrm{C}$. Transwell plates $(0.4 \mu \mathrm{m}$ pore; Corning, Inc.) were used for co-culture, with hDPCs or HaCaT cells seeded on the 6-well plate and hUCB-MSCs seeded in the 6-well inserts. Following $48 \mathrm{~h}$ co-culture, the inserts were removed in accordance with the 
experimental schedule to isolate hUCB-MSCs. To determine cell viability, hDPCs or HaCaT cells alone seeded in 6-well plates at a density of $2 \times 10^{5}$ cells $/ \mathrm{ml}$ were observed. Culture media were discarded, and cells were counted using the Cell Counting Kit-8 (CCK-8; Dojindo Molecular Technologies, Inc.) according to the manufacturer's instructions. Absorbance was measured at $450 \mathrm{~nm}$ using a SpectraMax i3x Multi-Mode Detection Platform (Molecular Devices, LLC).

Reverse transcription-quantitative polymerase chain reaction $(R T-q P C R)$. Total RNA was extracted from hDPCs and HaCaT cells using FavorPrep ${ }^{\mathrm{TM}}$ Tri-RNA reagent (Favorgen Biotech Corp.). First-strand cDNA synthesis was performed on the total RNA template using PrimeScript ${ }^{\mathrm{TM}}$ RT master mix (Takara Bio, Inc.). The resulting cDNA was subjected to qPCR using TOPreal ${ }^{\mathrm{TM}}$ qPCR $2 \mathrm{X}$ PreMIX containing SYBR $^{\circledR}$ (Enzynomics, Inc.) with a CFX-96 Touch $^{\mathrm{TM}}$ Real-Time PCR detection system (Bio-Rad Laboratories, Inc.). The thermocycling conditions were as follows: $10 \mathrm{~min}$ at $95^{\circ} \mathrm{C}$, followed by 40 cycles of $95^{\circ} \mathrm{C}$ for $15 \mathrm{sec}, 60^{\circ} \mathrm{C}$ for $30 \mathrm{sec}$ and $72^{\circ} \mathrm{C}$ for $30 \mathrm{sec}$. Expression data were calculated from the cycle threshold $(\mathrm{Cq})$ value using the $2^{-\Delta \Delta \mathrm{Cq}}$ method (23). Oligonucleotides used for qPCR were as follows: Human autophagy-related protein $(A T G) 6$ forward, 5'-AACCTCAGC CGAAGACTGAA-3' and reverse, 5'-CCTCTAGTGCCAGCT CCTTT-3'; human ATG8 forward, 5'-CGCACCTTCGAA CAAAGA GT-3' and reverse, 5'-GACCATGCTGTGTCCG TTC-3'; human cathepsin D (CTSD) forward, 5'-CAAGTTCGA TGGCATCCTGG-3' and reverse, 5'-CGGGTGACATTCAGG TAG GA-3'; human lysosomal-associated membrane protein 1 (LAMPl) forward, 5'-CTTTCAAGGTGGAAGGTGGC-3' and reverse, 5'-GATAGTCTGGTAGCCTGCGT-3'; human peroxisome proliferator-activated receptor gamma coactivator $1 \alpha$ $(P G C-1 \alpha)$ forward, 5'-AGCGCCGTGTGATTTATGTC-3' and reverse, 5'-TGCGTCCACAAAAGTACAGC-3'; human $D K K-1$ forward, 5'-TTCCGAGGAGAAATTGAGGA-3' and reverse, 5'-CCTGAGGCACAGTCTGATGA-3'; human alkaline phosphatase liver/bone/kidney isozyme (ALPL) forward, 5'-CCT CCTCGGAAGACACTCTG-3' and reverse, 5'-AGACTGCGC CTGGTAGTTGT-3'; human $\beta$-catenin (CTNNB1) forward, 5'-GCCGGCTATTGTAGAAGCTG-3' and reverse, 5'-GAG TCCCAAGGAGACCTTCC-3'.

Vascular endothelial growth factor (VEGF) concentration. hDPC culture media was collected following each experiment, and the VEGF levels in the supernatant were quantified using a Human VEGF Quantikine ELISA Kit (cat. no. DVE00; R\&D Systems, Inc.) according to the manufacturer's protocol. Absorbance was measured at $450 \mathrm{~nm}$ using a SpectraMax i3x Multi-Mode Detection Platform.

Propidium iodide (PI) fluorescence images. Following stimulation under each condition, $\mathrm{HaCaT}$ cells were washed with PBS and stained with $10 \mu \mathrm{g} / \mathrm{ml}$ PI for $5 \mathrm{~min}$ at $37^{\circ} \mathrm{C}$, and fluorescent images were observed under a DP70 fluorescence microscope with DP Controller software (DP-BSV ver. 03.03; Olympus Optical Co., Ltd.).

Western blot analysis. Mouse skin tissues or treated cells were harvested and washed twice with PBS at $4^{\circ} \mathrm{C}$. Protein was collected by lysing the cells in $200 \mu 1$ ice-cold PRO-PREP ${ }^{\mathrm{TM}}$ buffer (iNtRON Biotechnology, Inc.). The protein concentration was evaluated using a Bicinchoninic Acid Protein Assay kit (Thermo Fisher Scientific, Inc.). Aliquots of protein (30 $\mu \mathrm{g} /$ lane) were separated by $10-15 \%$ SDS-PAGE and transferred onto PVDF membranes. The membranes were blocked $5 \%$ non-fat milk in TBS-T for $2 \mathrm{~h}$ at room temperature and incubated with primary antibodies against poly (ADP-ribose) polymerase (PARP; cat. no. 9532S; 1:1,000; Cell Signaling Technology, Inc.), Bcl-2 (cat. no. SC-492; 1:1,000; Santa Cruz Biotechnology, Inc.), Bax (cat. no. SC-526; 1:1,000; Santa Cruz Biotechnology, Inc.), pro-caspase-3 (cat. no. ab32499; 1:1,000; Abcam), cleaved-caspase-3 (cat. no. ab2302; 1:1,000; Abcam), pro-caspase-9 (cat. no. ab138412; 1:1,000, Abcam), cleaved-caspase-9 (cat. no. ab2302; 1:1,000; Abcam), $\beta$-catenin (cat. no. ab16051; 1:1,000; Abcam), DKK-1 (cat. no. ab61034; 1:1,000; Abcam), LC3BI/II (cat. no. PM036; 1:1,000; MBL International Co.), p62 (cat. no. PM045; 1:1,000; MBL International Co.), Beclin1 (cat. no. PD017; 1:1,000; MBL International Co.), LAMP1 (cat. no. sc-20011; 1:1,000; Santa Cruz Biotechnology, Inc.), transcription factor C/EBP homologous protein (CHOP; cat. no. sc-7351; 1:1,000; Santa Cruz Biotechnology, Inc.), protein kinase R (PKR)-like endoplasmic reticulum kinase (PERK; cat. no. sc-377400; 1:1,000; Santa Cruz Biotechnology, Inc.) and $\beta$-actin (cat. no. sc-47778; 1:1,000, Santa Cruz Biotechnology, Inc.) at $4^{\circ} \mathrm{C}$ overnight. Goat anti-rabbit (cat. no. BA-1000; Vector Laboratories, Inc.) and anti-mouse (cat. no. WB-2000; Vector Laboratories, Inc.) were used as secondary antibodies $(1: 10,000)$ and incubated for $2 \mathrm{~h}$ at room temperature. The blots were analyzed by densitometry using ImageJ 1.44 software (National Institutes of Health).

Determination of mitochondrial mass and mitochondrial membrane potential (MMP). Mitochondrial mass (MitoTracker Green FM; cat. no. M7514; 100 nM; excitation/emission, 490/525 nm; Invitrogen; Thermo Fisher Scientific, Inc.) and MMP (Tetramethylrhodamine, Ethyl Ester, Perchlorate; cat. no. T669; 200 nM excitation/emission, 549/582 nm; Invitrogen Thermo Fisher Scientific, Inc.) were measured in $\mathrm{HaCaT}$ cells based on the fluorescence levels following staining with selective dyes at $37^{\circ} \mathrm{C}$ for $30 \mathrm{~min}$. HaCaT cells were washed once with PBS and promptly evaluated using a SpectraMax i3x Multi-Mode Detection Platform.

Statistical analysis. Data are presented as the mean \pm standard deviation (SD) of three independent experiments. Statistical analyses were performed using SPSS 17.0 (SPSS, Inc.). Differences between two groups were evaluated using a paired t-test. For multiple comparisons, one-way ANOVA followed by Tukey's multiple comparisons test was used. $\mathrm{P}<0.05$ was considered to indicate a statistically significant difference.

\section{Results}

hUCB-MSCs prevent hair regression induced by topical treatment with Dex. The schedule of the animal experiments are presented Fig. 1A. To determine whether hUCB-MSCs extended the anagen phase of the hair cycle, the growth of hair shafts in all groups was visually observed on day 16 
A Experimental schedule
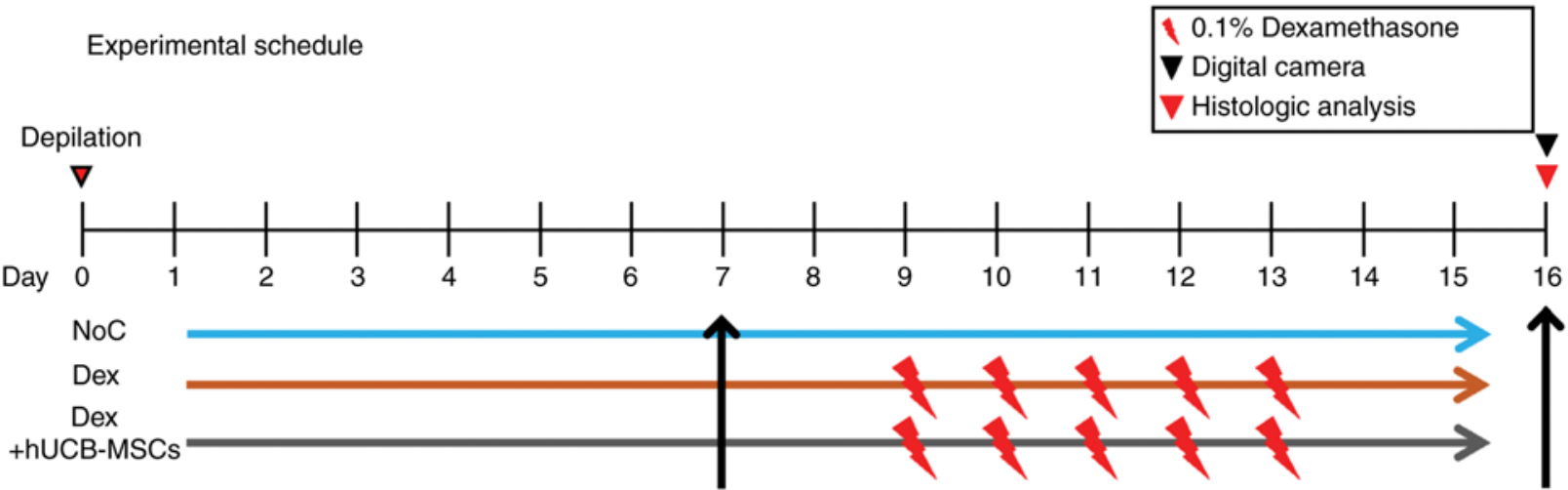

hUCB-MSCs, saline

intradermal injection

B

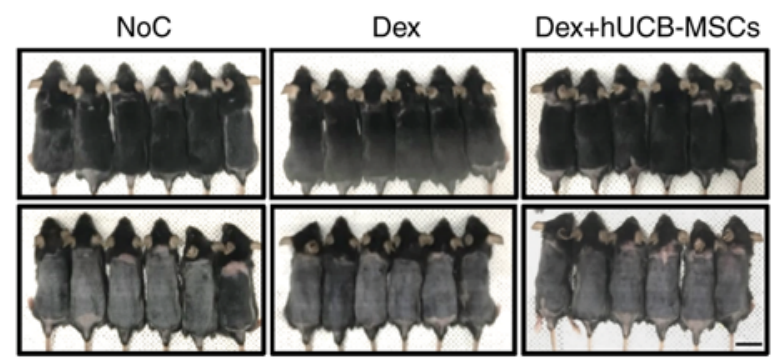

C

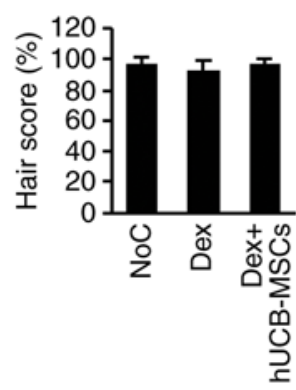

D

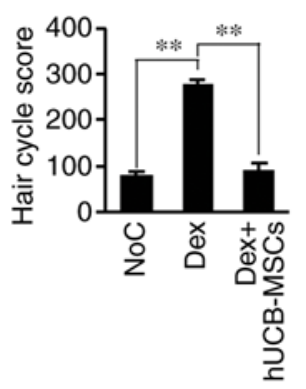

\section{G}

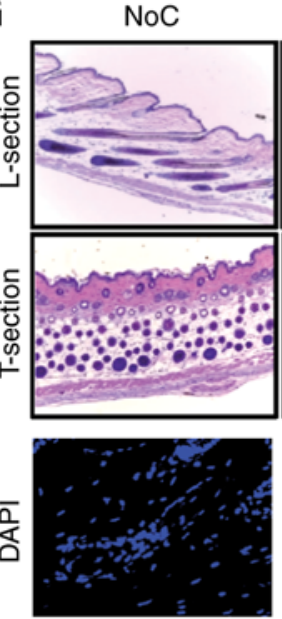

Dex

Dex+hUCB-MSCs
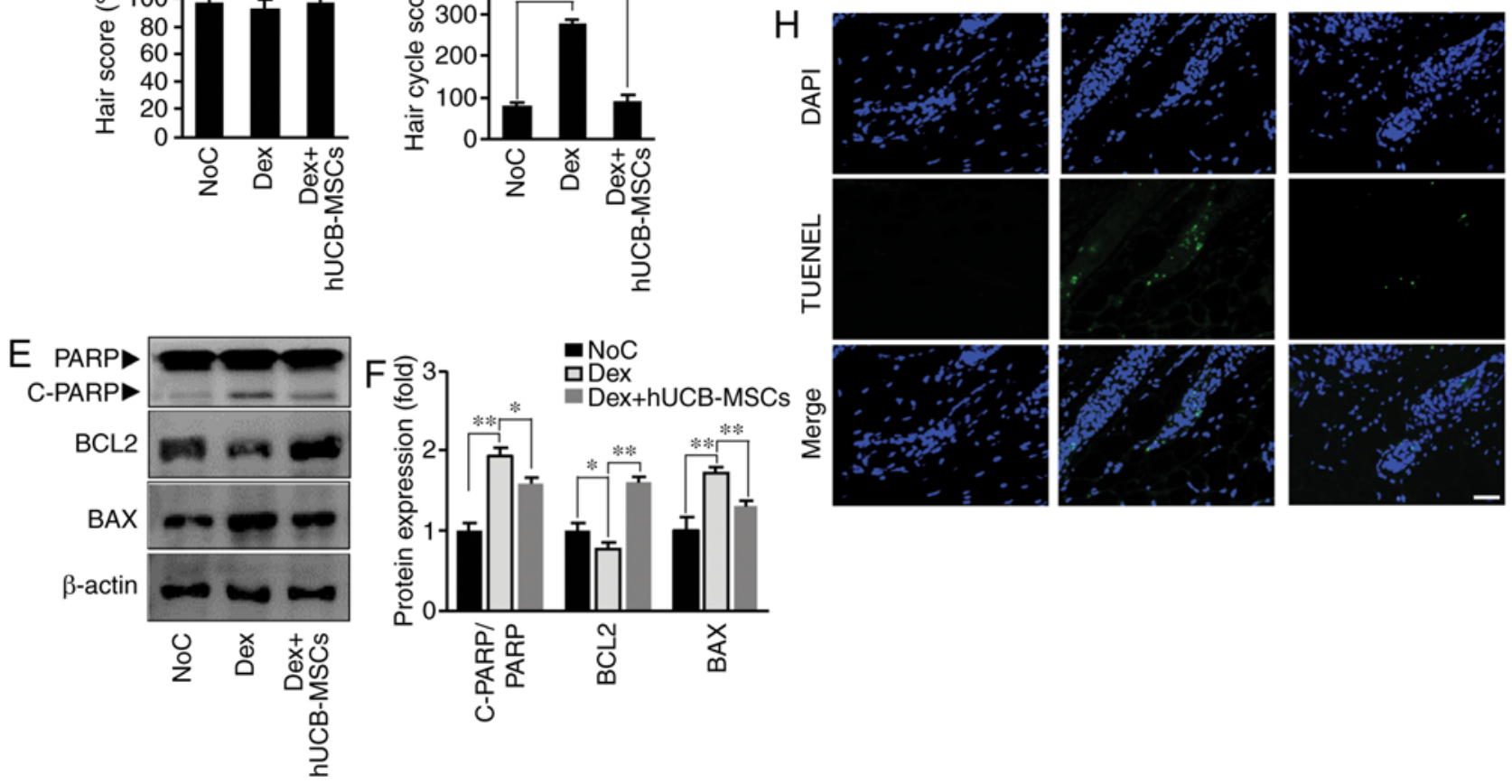

Figure 1. hUCB-MSCs prevent the catagen phase in C57BL/6 mice. (A) Experimental schedule. On day 7 of tape depilation, the NoC group was treated with $800 \mu 1$ saline; $1.25 \times 10^{5}$ cells/ml of hUCB-MSCs were injected into the intradermal site in the Dex + hUCB-MSCs group, and the Dex group was not treated. From day 9 to $13,1 \mathrm{ml}$ dexamethasone $(0.1 \%)$ was topically administered to the Dex and Dex + hUCB-MSCs groups. On day 16 , all animals were sacrificed. (B) The dorsal skin was photographed on day 16 (upper panel, pre-depilation; lower panel, post-depilation). Scale bar, $2.5 \mathrm{~cm}$. (C) Quantification of the hair score. Data are expressed as the mean \pm SD. (D) HCS on day 16 post-depilation. Higher HCS indicates the progression of catagen. (E) Western blot analysis of PARP, Bcl-2 and Bax levels in dorsal tissue from the three groups. (F) Densitometry analysis if protein expression. (G) Sections of the dorsal skin. L-section, longitudinal section; T-section, transverse section. Scale bar, $100 \mu \mathrm{m}$. (H) TUNEL staining. Representative images of TUNEL staining (green fluorescence, middle panel). DAPI was used as a counterstain (upper panel), and merged pictures are presented in the bottom panel. Scale bar, $25 \mu \mathrm{m}$. ${ }^{*} \mathrm{P}<0.05,{ }^{* *} \mathrm{P}<0.01 \mathrm{vs}$. Dex. NoC, normal control; Dex, dexamethasone; HCS, hair cycle score; hUCB-MSCs, human umbilical cord blood-derived mesenchymal stem cells; PARP, poly (ADP-ribose) polymerase.

after depilation (Fig. 1B, upper row). Hair cycle stage was determined by measuring the skin color status, and the results revealed diffuse darkening of the dorsal skin 16 days after removing hair (Fig. 1B, lower row). However, no significant differences were observed among the Con, Dex and Dex + hUCB-MSCs groups (Fig. 1B and C). 
A
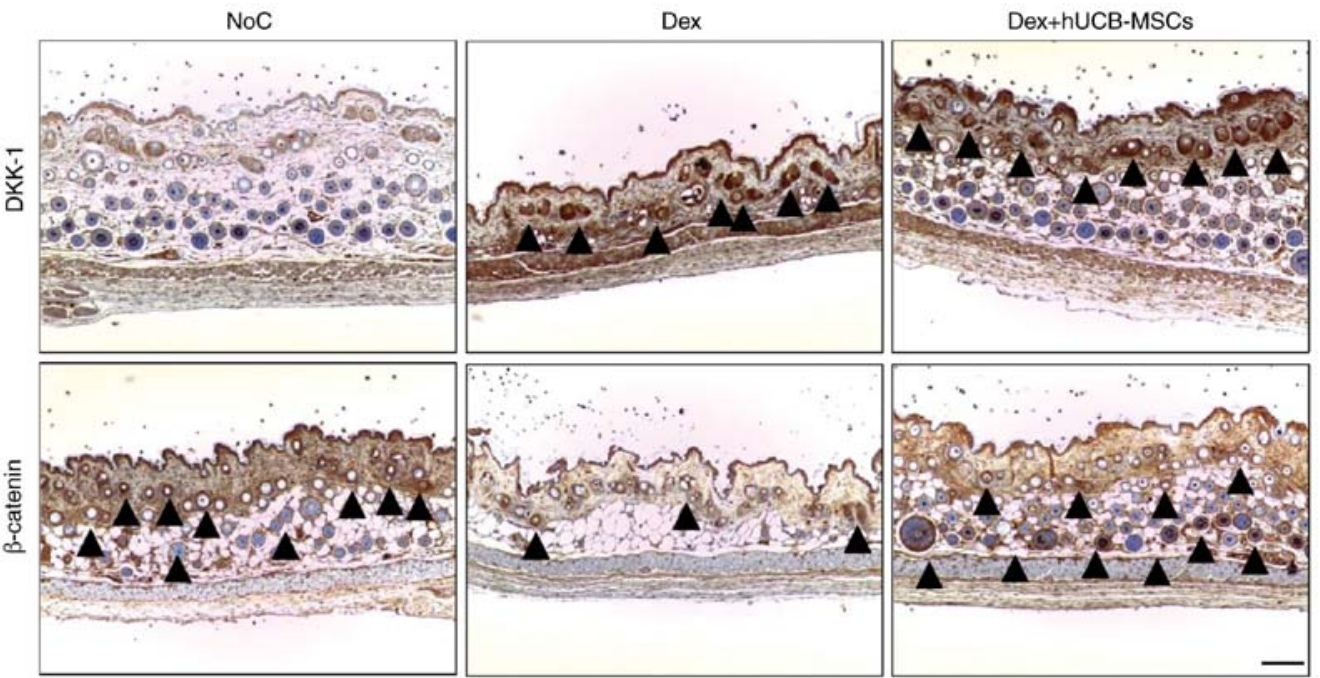

B
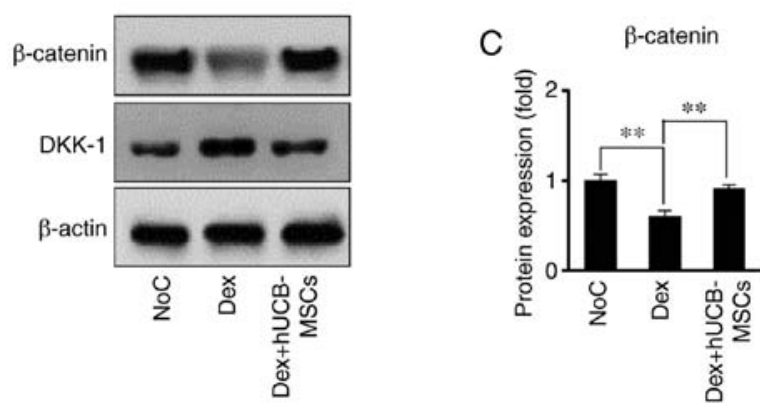

DKK-1

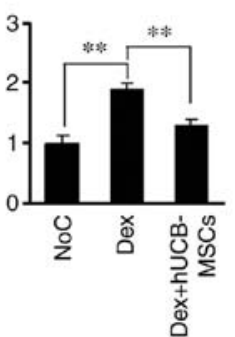

Figure 2. Effects of hUCB-MSCs on DKK-1 and $\beta$-catenin expression in mouse skin hair follicles. (A) Representative images of immunohistochemistry. Protein location was observed using anti-DKK-1 (upper panel) and anti- $\beta$-catenin (lower panel) antibodies. Black arrow heads indicate a positive reaction. Scale bar, $100 \mu \mathrm{m}$. (B) Western blot analysis of DKK-1 and $\beta$-catenin levels in dorsal skin tissue in the three groups. (C) Bar diagram of densitometry analysis ${ }^{* * *} \mathrm{P}<0.01$ vs. Dex. NoC, normal control; Dex, dexamethasone; hUCB-MSCs, human umbilical cord blood-derived mesenchymal stem cells; DKK-1, Dickkopf WNT signaling pathway inhibitor 1 .

Dorsal skin was harvested for histological analysis to determine whether hUCB-MSCs prolonged anagen (Fig. 1G, longitudinal section). Hair follicles treated with hUCB-MSCs maintained a longer anagen phase compared with the Dex group. The Dex + hUCB-MSCs group also maintained a higher hair follicle number compared with the Dex group (Fig. 1G, transverse section). In addition, the HCS in the Dex + hUCB-MSC group was lower compared with that in the Dex group, indicating that hUCB-MSCs prevented Dex-modulated transition to catagen (Fig. 1D).

The present study also investigated histological changes following hair regression to confirm the protective effect of hUCB-MSCs. Mice treated with Dex exhibited severe histopathological degeneration, which was determined by TUNEL staining, whereas injection with hUCB-MSCs ameliorated these changes (Fig. 1H). This suggested that Dex-induced apoptosis in hair follicles may be responsible for hair regression. In addition, the present study examined whether hUCB-MSCs regulated PARP-dependent cell death. Dex induced an increase in the expression of the pro-apoptotic protein Bax and cleavage of PARP, as well as a decrease in the expression of the anti-apoptotic protein Bcl-2. By contrast, hUCB-MSCs counteracted PARP-dependent apoptosis compared with the Dex group (Fig. 1E and F). These results indicated that hUCB-MSC transplantation ameliorated follicular cell death by reducing hair follicle apoptosis.
hUCB-MSCs prevent DKK-1 increase and $\beta$-catenin decrease in hair follicles. Mouse dorsal skin tissue samples were used to investigate whether Dex-stimulated hair regression was associated with DKK-1 and $\beta$-catenin expression. Compared with the NoC group, DKK-1 expression increased in the Dex group (Fig. 2A, upper panel). However, hUCB-MSCs significantly attenuated this upregulated DKK-1 expression in hair follicles. In addition, hUCB-MSC treatment significantly changed the expression of $\beta$-catenin, which is a positive regulator of hair growth, compared with the Dex group (Fig. 2A, lower panel). The levels of DKK-1 and $\beta$-catenin were significantly reversed in mouse skin tissues; in the Dex group, DKK-1 expression was increase and $\beta$-catenin expression was decreased compared with the control group, whereas in the Dex + hUCB-MSCs group, DKK-1 expression was decrease and $\beta$-catenin expression was increased compared with the Dex group (Fig. 2B and C).

hUCB-MSCs reduce the deposition of p62 and increase LC3 expression in hair follicles. To investigate the role of autophagy in hUCB-MSC intra-dermal transplantation and hair regression in mouse skin tissue, the expression levels of the negative regulator p62 and the positive regulator LC3, which are key markers of autophagy regulation $(24,25)$, were determined. The results demonstrated that Dex-induced hair regression increased the deposition of p62 in mouse hair follicles in the Dex group (Fig. 3A). However, the accumulation of p62 was 

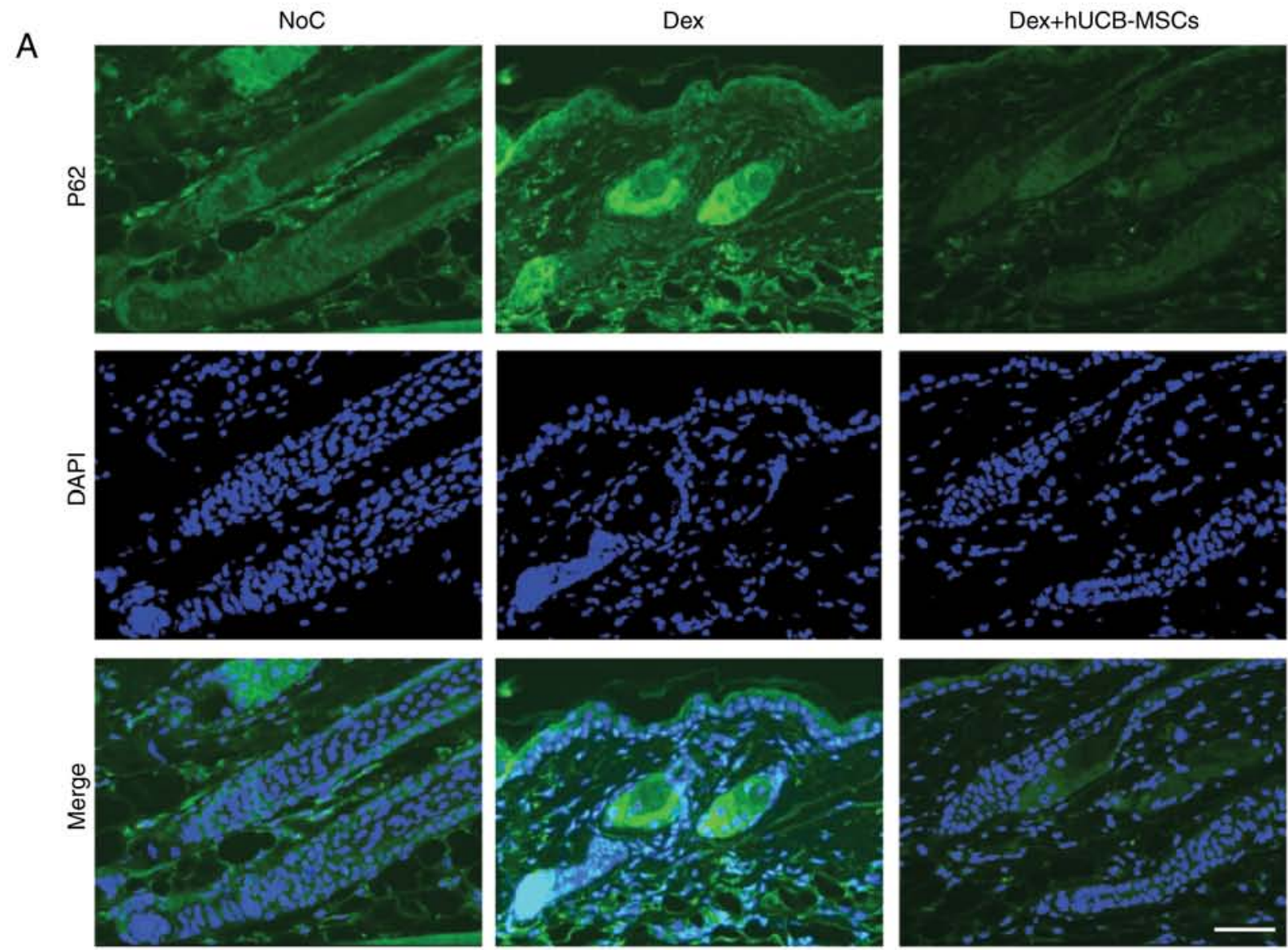

B
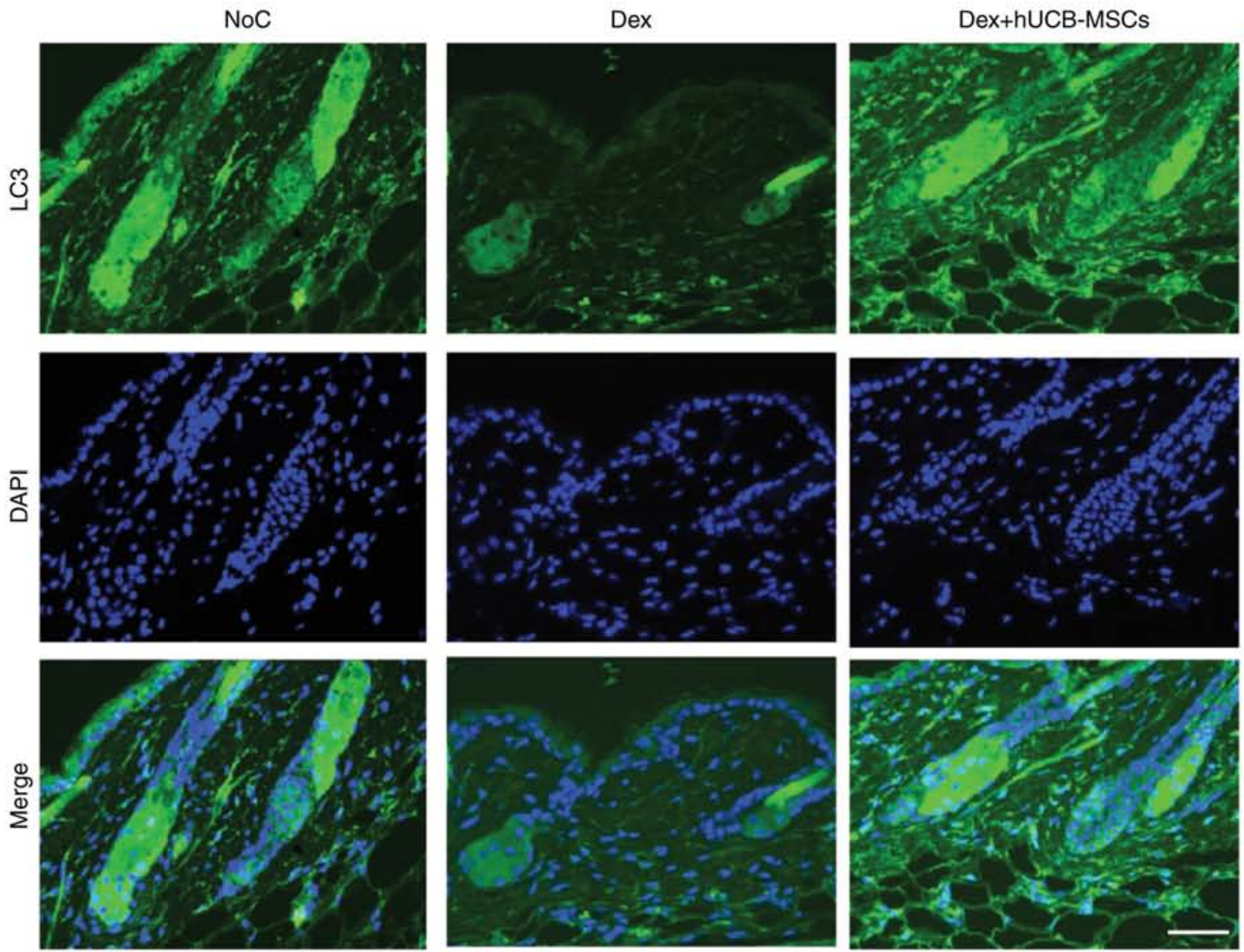

Figure 3. Effects of hUCB-MSCs on LC3 and p62 expression in mouse skin hair follicles. (A and B) Representative immunofluorescence images. Protein localization was observed using (A) anti-p62 and (B) anti-LC3 antibodies. DAPI was used as a counterstain. Scale bar, $100 \mu \mathrm{m}$. 

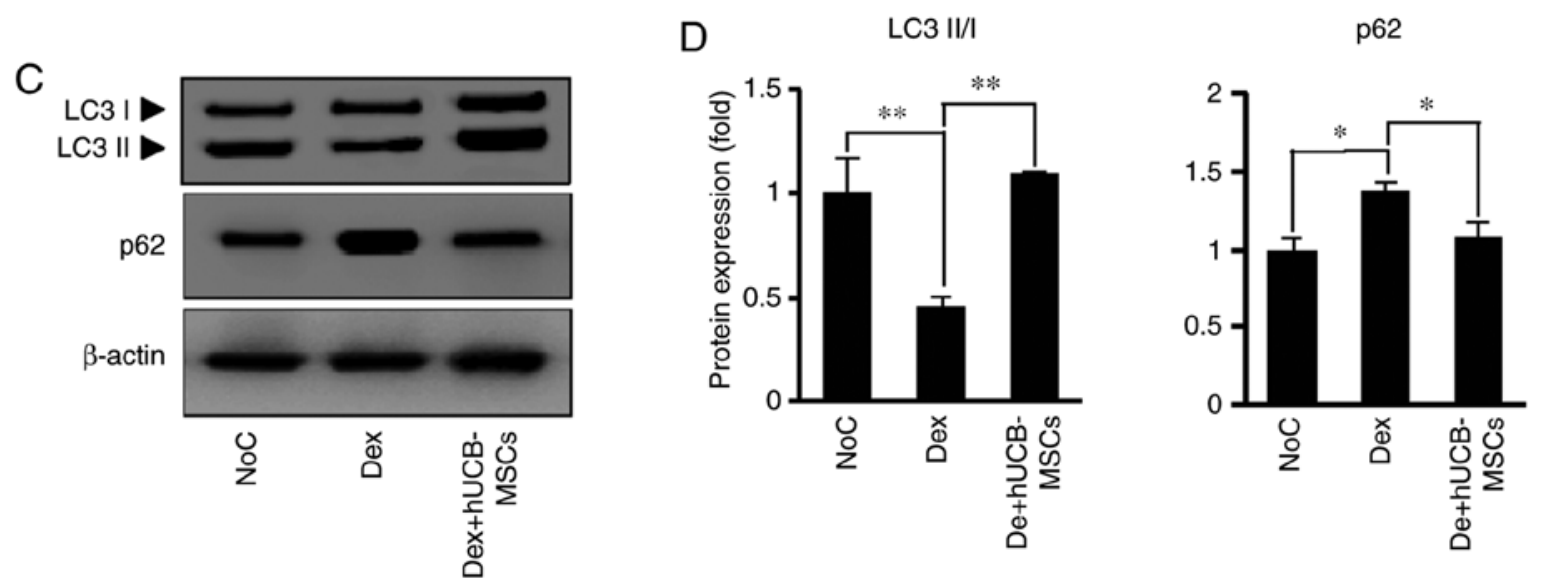

Figure 3. Continued. (C) Western blot analysis of LC3 and p62 levels in dorsal skin tissues of the 3 groups. (D) Bar diagram of densitometry analysis. ${ }^{*} \mathrm{P}<0.05,{ }^{* *} \mathrm{P}<0.01$ vs. Dex. NoC, normal control; Dex, dexamethasone; hUCB-MSCs, human umbilical cord blood-derived mesenchymal stem cells; LC3, microtubule-associated protein 1 light chain $3 \beta$.

diminished in the Dex + hUCB-MSCs group in a manner similar to that of the NoC group.

In the present study, LC3 expression in the Dex + hUCB-MSCs group significantly recovered compared with the Dex group, and the recovery level was similar to that of the NoC group (Fig. 3B). Of note, LC3II was significantly increased in the Dex + hUCB-MSCs group compared with the Dex group; by contrast, p62 was increased in the Dex group but significantly decreased in the Dex + hUCB-MSCs group (Fig. 3C and D). These results demonstrated that Dex-induced hair follicle damage was likely due to malfunction of the autophagic process.

hUCB-MSCs reduce the inhibitory effects of Dex on cell viability and decrease DKK-1 expression in hDPCs. Analysis of the viability of hDPCs stimulated by Dex (25, 50 and $100 \mu \mathrm{M}$ ) for $48 \mathrm{~h}$ revealed that cell viability decreased in a concentration-dependent manner compared with the control group (Fig. 4B). To confirm the effects of hUCB-MSCs on the inhibition of hDPC viability by Dex, hUCB-MSCs were co-cultured with hDPCs using a Transwell plate. Co-culture with hUCB-MSC not only increased the viability of hDPCs, but also partially restored the reduction of hDPC viability induced by Dex (Fig. 4C).

VEGF has previously been identified as a key mediator of hair generation and growth (26). Therefore, growth factor secretion in the presence or absence of Dex and hUCB-MSCs was compared in the present study using ELISA. VEGF secretion following treatment with $100 \mu \mathrm{M}$ Dex was significantly decreased compared with the control group (Fig. 4D). However, co-treatment with Dex and hUCB-MSCs demonstrated that hUCB-MSCs counteracted the inhibitory effect of Dex on VEGF secretion. To evaluate the amount of VEGF secreted from hUCB-MSCs, secretion of VEGF in hUCB-MSCs alone was measured in the culture medium; the concentration of VEGF obtained from hUCB-MSCs was $152.73 \pm 12.7 \mathrm{pg} / \mathrm{ml}$. The results indicated that the amount of VEGF released from hDPCs in the Dex + hUCB-MSCs group $(\sim 117 \mathrm{pg} / \mathrm{ml})$ was higher compared with that in the Dex group, even when the amount of VEGF secreted by hUCB-MSCs was excluded.
To investigate whether hUCB-MSCs modulated the expression of genes associated with hair growth in hDPCs, the expression levels of $D K K-1, C T N N B 1$ and $A L P L$ mRNA, as well as DKK-1 protein (27), were analyzed in the presence or absence of Dex. DKK-1 expression was decreased at the mRNA and protein levels in the Dex + hUCB-MSCs group compared with the Dex group (Fig. 4E-G). Additionally, $C T N N B 1$ and $A L P L$ mRNA expression increased in the hUCB-MSC co-culture groups in the presence and absence of Dex. These results suggested that hUCB-MSCs may protect against Dex stimulation-induced cell damage in hDPCs.

hUCB-MSCs protect HaCaT cells against apoptosis Dex-induced. To determine whether Dex induced keratinocyte cell death directly, $\mathrm{HaCaT}$ cells were treated with different concentrations of Dex for $48 \mathrm{~h}$, and the cell viability was measured. Cell viability was decreased compared with the Con group by treatment with 25, 50 and $100 \mu \mathrm{M}$ Dex (Fig. 5A). To confirm the effect of hUCB-MSCs on the inhibitory effects of Dex on HaCaT proliferation, hUCB-MSCs were co-cultured with $\mathrm{HaCaT}$ using a Transwell plate. Of note, co-culture with hUCB-MSCs increased HaCaT cell viability and rescued the Dex-reduced viability during co-culture of hUCB-MSCs in the presence of Dex (Fig. 5B). To investigate whether the inhibitory effects of Dex on HaCaT viability were apoptosis-dependent, cells were stained with PI and the expression of Bcl-2, Bax, caspase-9, caspase-3 and PARP proteins was analyzed. PI-positive expression was increased in the Dex group, which was counteracted by hUCB-MSCs in the Dex + hUCB-MSCs group (Fig. 5C). To verify apoptosis activation, PARP-dependent apoptosis upon Dex treatment was analyzed using western blotting. The cleaved form of caspase-9, caspase-3, PARP and the $\mathrm{Bax} / \mathrm{Bcl}-2$ ratio were increased in the Dex group, whereas hUCB-MSC co-culture counteracted the upregulation of apoptosis (Fig. 5D and E).

Mitochondrial biogenesis markers, mitochondrial mass and MMP were evaluated to elucidate the protective effect of hUCB-MSCs on Dex-induced cell death. PGC-1 $\alpha$ transcription was upregulated by hUCB-MSCs (Fig. 5F). Decreased mitochondrial mass and MMP were counteracted in $\mathrm{HaCaT}$ cells by the presence of hUCB-MSCs (Fig. 5G and H). These 

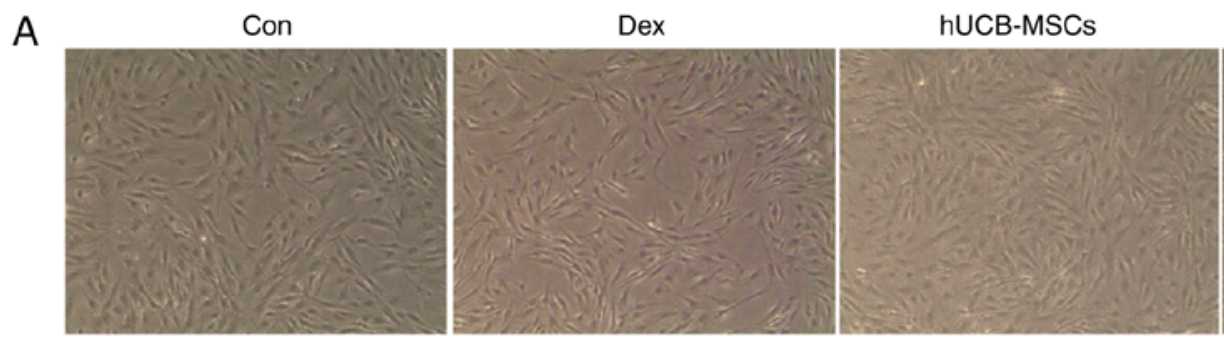

Dex+hUCB-MSCs
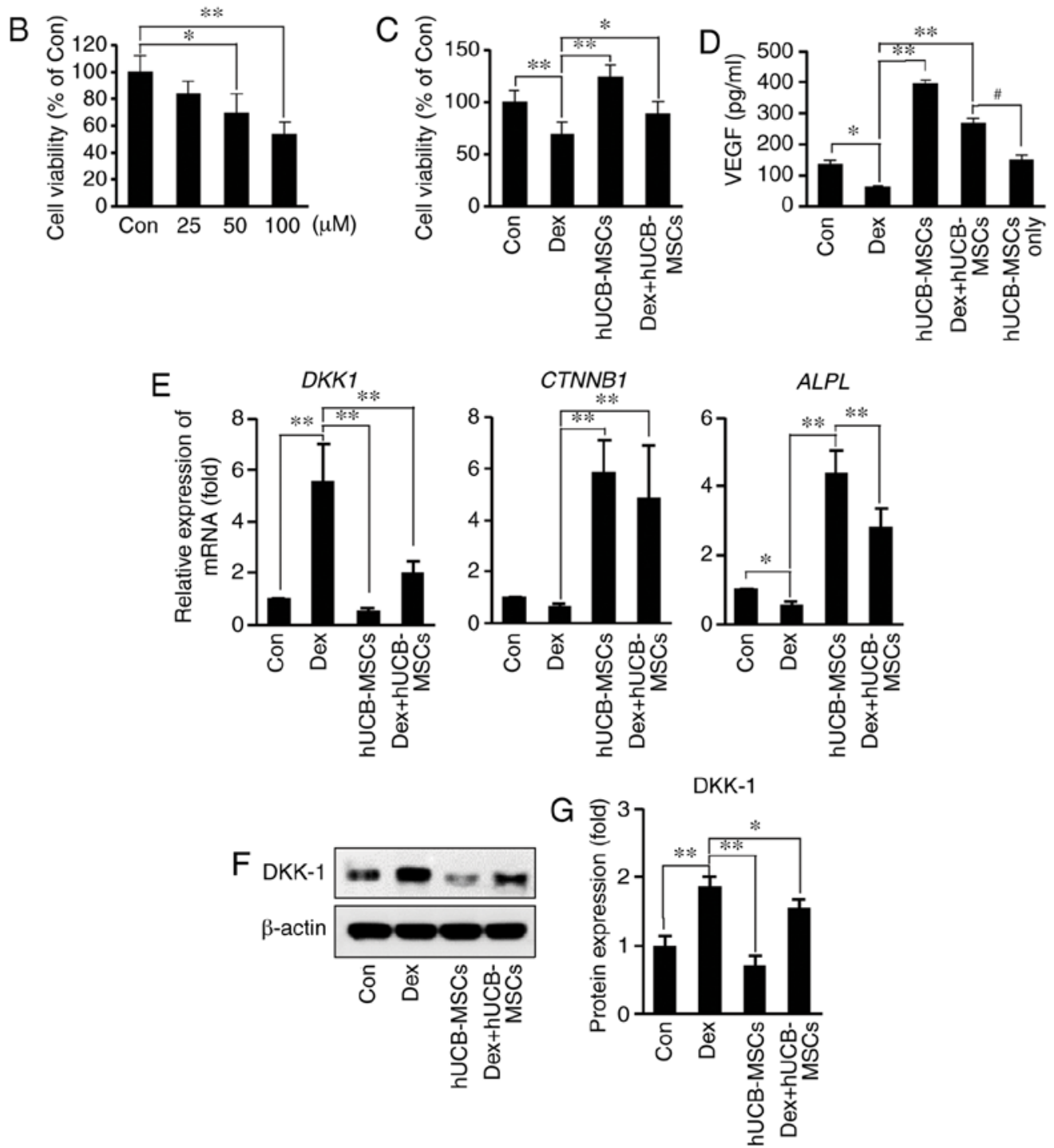

Figure 4. Effects of hUCB-MSC co-culture on viability, VEGF secretion, mRNA and protein expression in hDPCs following Dex stimulation. (A) Representative images of hDPCs. Cell density was decreased by Dex stimulation; hUCB-MSCs counteracted the reduction. Scale bar, $50 \mu \mathrm{m}$. (B) hDPCs were treated with 25-100 $\mu \mathrm{M}$ Dex for $48 \mathrm{~h}$. Cell viability was determined via MTT assay. ${ }^{*} \mathrm{P}<0.05,{ }^{* *} \mathrm{P}<0.01$ vs. Con. (C) Effects of hUCB-MSCs on the viability of hDPCs stimulated with $100 \mu \mathrm{M}$ Dex for $48 \mathrm{~h}$ in the presence or absence of hUCB-MSCs. Cell viability was determined via MTT assay. ${ }^{*} \mathrm{P}<0.05,{ }^{* *} \mathrm{P}<0.01 \mathrm{vs}$. Dex. (D) Secretory concentration of VEGF. hDPCs were stimulated with $100 \mu \mathrm{M}$ Dex for $48 \mathrm{~h}$ in the presence or absence of hUCB-MSCs, VEGF concentration in the culture supernatant was evaluated using ELISA. ${ }^{\mathrm{P}} \mathrm{P}<0.05,{ }^{* *} \mathrm{P}<0.01$ vs. Dex,; ${ }^{*} \mathrm{P}<0.05$ vs. Dex + hUCB-MSCs. (E) Expression of $D K K-1, C T N N B 1$ and ALPL mRNA was determined by reverse transcription-quantitative PCR in hDPCs stimulated with $100 \mu \mathrm{M}$ Dex for $24 \mathrm{~h}$ in the presence or absence of hUCB-MSCs. ${ }^{*} \mathrm{P}<0.05,{ }^{* * *} \mathrm{P}<0.01$ vs. Dex. (F) Western blot analysis of DKK-1 levels in hDPCs stimulated with $100 \mu \mathrm{M}$ Dex for $24 \mathrm{~h}$ in the presence or absence of hUCB-MSCs. (G) Bar diagram of densitometry analysis. ${ }^{*} \mathrm{P}<0.05,{ }^{* *} \mathrm{P}<0.01$ vs. Dex. hDPCs, human dermal papilla cells; Con, control; Dex, dexamethasone; hUCB-MSC, human umbilical cord blood-derived mesenchymal stem cell; VEGF, vascular endothelial growth factor; DKK-1, Dickkopf WNT signaling pathway inhibitor 1; CTNNB1, $\beta$-catenin; $A L P L$, human alkaline phosphatase liver/bone/kidney isozyme.

results suggested that co-culture with hUCB-MSCs may protect the quantity and function of mitochondria. In addition, Dex stimulation may induce keratinocyte death directly via apoptosis, and hUCB-MSCs may exert anti-apoptotic effects on Dex stimulation.
hUCB-MSCs regulate autophagy and decrease unfolding protein response (UPR) in HaCaT cells. To investigate whether autophagy regulation and UPR were influenced by Dex stimulation and to determine the effects of hUCB-MSCs in this context, changes in autophagy-related genes and ER stress markers at 

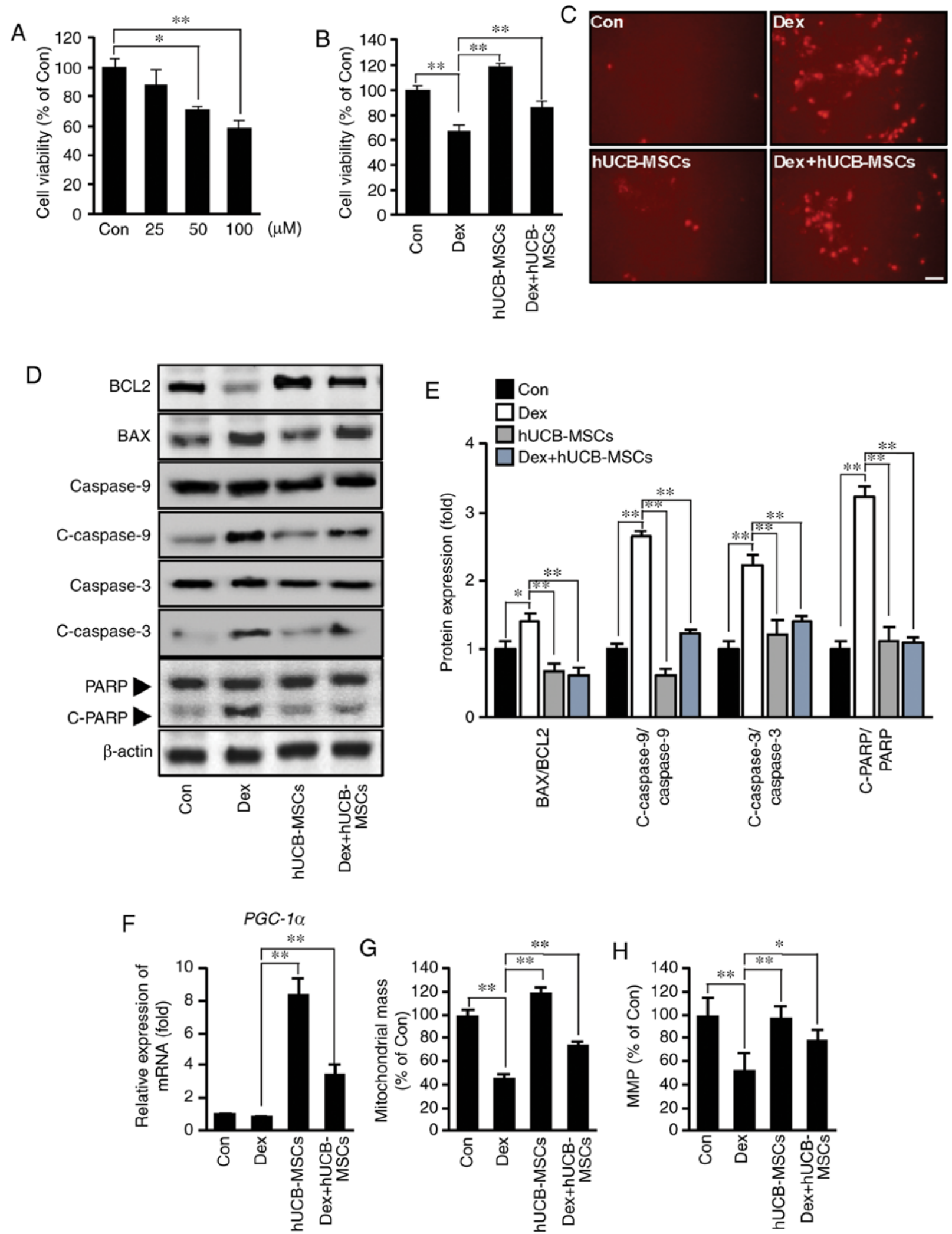

Figure 5. Effects of hUCB-MSC co-culture on apoptosis and mitochondria biogenesis after Dex stimulation in HaCaT cells. (A) HaCaT cells were treated with $25-100 \mu \mathrm{M}$ Dex for $48 \mathrm{~h}$. Cell viability was determined using MTT assay. ${ }^{*} \mathrm{P}<0.05,{ }^{* *} \mathrm{P}<0.01$ vs. Con. (B) Effects of hUCB-MSCs on the viability of $\mathrm{HaCaT}$ cells stimulated with $100 \mu \mathrm{M}$ Dex for $48 \mathrm{~h}$ in the presence or absence of hUCB-MSCs. Cell viability was determined via MTT assay. ${ }^{* *} \mathrm{P}<0.01$ vs. Dex. (C) Representative images of $\mathrm{HaCaT}$ cells stained with PI. Positive reactions were increased by Dex stimulation, whereas hUCB-MSCs counteracted apoptotic reactions. Scale bar, $50 \mu \mathrm{m}$. (D) Western blot analysis of Bcl-2, Bax, caspase-9, C-caspase-9, caspase-3, C-caspase-3 and PARP levels in HaCaT cells stimulated with $100 \mu \mathrm{M}$ Dex for $48 \mathrm{~h}$ in the presence or absence of hUCB-MSCs. (E) Bar diagram of densitometry analysis. ${ }^{* *} \mathrm{P}<0.01 \mathrm{vs}$. Dex. (F) $P G C-1 \alpha \mathrm{mRNA}$ expression was determined using reverse transcription-quantitative PCR in HaCaT cells stimulated with $100 \mu \mathrm{M}$ Dex for $24 \mathrm{~h}$ in the presence or absence of hUCB-MSCs. ${ }^{* *} \mathrm{P}<0.01$ vs. Dex. (G) Mitochondrial mass was measured using MitoTracker fluorescence signals. ${ }^{* *} \mathrm{P}<0.01$ vs. Dex. (H) MMP was measured under the indicated conditions using the MMP-sensitive probe. ${ }^{*} \mathrm{P}<0.05,{ }^{* *} \mathrm{P}<0.01$ vs. Dex. Con, control; Dex, dexamethasone; hUCB-MSC, human umbilical cord blood-derived mesenchymal stem cell; PI, propidium iodide; C, cleaved; PARP, poly (ADP-ribose) polymerase; MMP, mitochondrial membrane potential; $P G C$-1 $\alpha$, peroxisome proliferator-activated receptor gamma coactivator $1 \alpha$. 

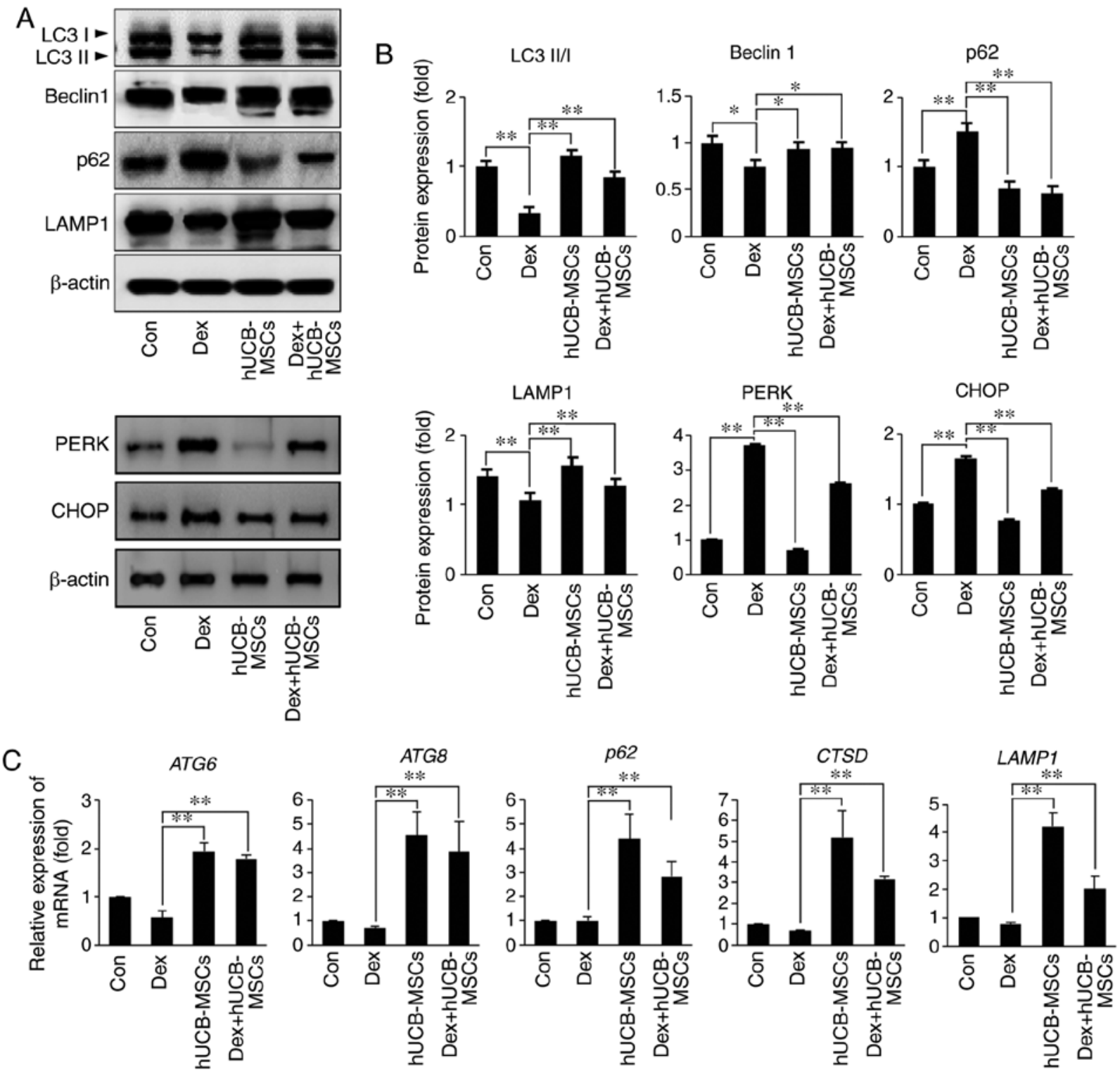

Figure 6. Effects of hUCB-MSC co-culture on autophagy and ER stress in HaCaT cells following Dex stimulation. (A) HaCaT cell lysates were immunoblotted with antibodies against LC3I/II, Beclin1, p62, LAMP1, PERK, CHOP or $\beta$-actin. (B) Densitometry analysis results. ${ }^{* *} \mathrm{P}<0.01$ vs. Dex group. (C) Transcript levels of autophagy-related genes were determined by reverse transcription-quantitative PCR. ${ }^{* *} \mathrm{P}<0.01$ vs. Dex group. LC 3 , microtubule-associated protein 1 light chain $3 \beta$ Con, control; Dex, dexamethasone; hUCB-MSC, human umbilical cord blood-derived mesenchymal stem cell; LAMP1, human lysosomal-associated membrane protein 1; CHOP, transcription factor C/EBP homologous protein; PERK, protein kinase R (PKR)-like endoplasmic reticulum kinase; ATG, autophagy-related protein.

the protein and mRNA levels were quantified in HaCaT cells. Co-culture with hUCB-MSCs increased the conversion of LC3-I to LC3-II compared with that in the Dex group (Fig. 6A and B). In addition, Beclin1 and p62 expression levels indicated that autophagy regulation increased in the presence of hUCB-MSCs. The expression of LAMP-1, which degrades macromolecules from the cytoplasm through autophagy (28), was significantly increased in cells co-cultured with hUCB-MSCs compared with the Dex group. Similarly, the transcription levels of autophagy-related genes ATG6, ATG8, p62, CTSD and LAMP1 were increased in the hUCB-MSC co-culture groups compared with the control and Dex groups (Fig. 6C). Transcription of p62 was increased by hUCB-MSCs, whereas the p62 protein level was decreased in cells treated with hUCB-MSCs (Fig. 6A and B). To understand the association between UPR, cell damage and autophagy, the expression levels of PERK (an ER membrane sensor) and CHOP (a command executor in ER stress), which are ER stress markers, we observed. PERK and CHOP protein expression levels were increased by Dex treatment, indicating that unfolding proteins accumulated in $\mathrm{HaCaT}$ cells, resulting in ER stress. However, hUCB-MSC co-culture decreased the ER stress markers in the presence or absence of Dex treatment. These results suggested that hUCB-MSC treatment enhanced the autophagic flux and reduced the accumulation of unfolding proteins in keratinocytes.

\section{Discussion}

Dermal papilla cells are small, elongated, rain droplet-shaped cells that extend from the epidermis into the dermis (29). 
Dermal cells with mesoderm-derived stem cell characteristics serve a pivotal role in the formation of hair follicle tissues, and their function is important in hair loss (30). Therefore, the possibility of treating hair loss using human-derived MSCs has been suggested in previous studies. Yoo et al (31) have demonstrated that dermal papilla-like tissues produced by mesenchymal stem cells exhibited novel hair follicle-inducing activity upon transplantation in athymic mice, suggesting that human stem cells from the bone marrow or the umbilical cord may be novel and usable sources of stem cells. Our previous study demonstrated that conditioned media from hUCB-MSCs contain various beneficial proteins such as growth factors and cytokines (32). In addition, as a precedent, the application of adipose tissue-derived stem cell-conditioned media containing paracrine factors has exhibited potential as a therapeutic alternative in female patients with alopecia (33). Paracrine factors from MSCs actively participate in anti-apoptotic mechanisms, as well as stimulation of proliferation and removal of toxic proteins $(34,35)$. These findings suggest that human-derived stem cells or their paracrine factors have a potential for hair loss treatment.

Topical administration of Dex causes hair follicle degeneration, thus indicating the clinical phenotype of the catagen phase (36). In addition, Dex application increased DKK-1 protein expression levels in human dermal papilla cells, which was followed by hair follicle apoptosis (37). DKK-1 acts as an antagonist of the Wnt/ $\beta$-catenin pathway, and secretory DKK-1 induces apoptosis of follicular keratinocytes (38). The Wnt/ $\beta$-catenin pathway acts as an inductive signal for hair growth by maintaining dermal papilla cells in the anagen phase (39). In the case of Wnt stimulation in dermal papilla cells, $\beta$-catenin (encoded by the CTNNB1 gene) expression increases, and $\beta$-catenin accumulates in the nuclei of dermal papilla during anagen, providing hair growth signals (39). These findings suggest that the $\beta$-catenin pathway may be a target for the development of drugs that activate hair growth by inducing anagen phase genes (40). In the present study, intradermal injection of hUCB-MSCs not only decreased DKK-1 expression in mouse skin, but also normalized $\beta$-catenin expression compared with that in the control group. Expression of ALPL, a prominent dermal papilla marker, is dependent on the hair cycle and is most highly expressed during the anagen phase; thus, it has been identified as a critical marker for hair growth promotion (41). The expression of the $A L P L$ gene was also increased in hUCB-MSC co-culture, indicating that hUCB-MSCs affected anagen maintenance via $\beta$-catenin signaling, as reported previously (42). Of note, apoptosis was decreased in the Dex + hUCB-MSC group compared with the Dex group in vivo. Furthermore, in vitro, hUCB-MSCs protected hDPCs against Dex-induced cell damage and decreased DKK-1 mRNA and protein expression levels. These results suggested that hUCB-MSCs may prevent hair catagen by suppressing DKK-1 expression. Although a previous study indicated that Dex increased DKK-1 secretion from hDPCs followed by outer root sheath apoptosis, this did not confirm that Dex directly influences keratinocyte cell death (37). The results of the present study demonstrated that Dex inhibited cell proliferation, followed by the induction of keratinocyte apoptosis. GC mainly binds GRs and influences cell proliferation or cell damage (43); although a number of GRs are located in skin keratinocytes (44), previous studies have identified the positive effects of Dex in atopic dermatitis and wound healing $(45,46)$. However, another study has reported that an elevated concentration of Dex decreased cell proliferation and induced apoptosis and/or necrosis in endothelial cells (47). In the present study, Dex reduced cell proliferation and induced apoptosis by increasing the $\mathrm{Bax} / \mathrm{Bcl}-2$ ratio, cleaved-caspase-3, cleaved-caspase-9 and cleaved PARP in keratinocytes.

Llambiand and Green (48) have reported that mitochondria-mediated apoptosis is mainly associated with the Bcl-2 family. Therefore, in the apoptosis pathway, the ratio of $\mathrm{Bax} / \mathrm{Bcl}-2$ and $\mathrm{Bax} / \mathrm{Bcl}$-extra large serve an important role, as do the expression levels of Bcl-2 family members (49). In the present study, hUCB-MSCs not only decreased the Bax/Bcl-2 ratio, but also increased the mRNA levels of $P G C-1 \alpha$, a master regulator of mitochondrial biogenesis (50). In addition, in the presence of hUCB-MSCs, mitochondrial mass and MMP were increased compared with the Dex group in HaCaT cells. These results suggested that hUCB-MSCs and their paracrine factors protected $\mathrm{HaCaT}$ cells against apoptosis and that there may be an underlying mechanism associated with mitochondrial function and biogenesis.

The role of autophagy as a degradative pathway is crucial in regenerative medicine. Induction of autophagy under pathological conditions or cell stress may serve as an adaptive and protective mechanism for cell survival $(51,52)$. In a previous study, genetic inhibition of follicular autophagic flux induced and enhanced premature hair keratinocyte apoptosis, suggesting that autophagic flux in anagen phase keratinocytes is important for the maintenance of the hair growth phase (53). In the present study, hUCB-MSCs transplanted in mice were involved in LC3 lipidation, which was suppressed by Dex. Additionally, hUCB-MSCs protected $\mathrm{HaCaT}$ cells against Dex-induced apoptosis and increased autophagy-related mRNA and protein expression. In particular, the expression levels of lysosomal markers were significantly increased in the presence of hUCB-MSCs in HaCaT cells. The autophagosome cargo protein p62 gathers intracellular constructs that have broken structures or expired functions (54). If the autophagy process has not matured or is interrupted by specific stimuli, the autophagosomes cannot fuse with the lysosome to generate the autophagolysosome; thus, unnecessary cellular components are not lysed, resulting in p62 accumulation (55). In the present study, hUCB-MSC transplantation effectively decreased p62 accumulation in mouse hair follicles. In addition, an increase in the lysosomal markers and a decrease in the ER stress markers was confirmed in the detached environment in vitro. These results suggested that hUCB-MSCs and their paracrine factors restored autophagy processes disrupted by GCs.

To the best of our knowledge, the current study is the first to demonstrate that hUCB-MSCs may prevent hair regression caused by Dex-induced hair follicular damage. Although other mechanisms underlying these protective effects remain to be determined, maintaining autophagic flux was identified as a crucial mechanism of anagen phase expansion during hair loss.

\section{Acknowledgements}

Not applicable. 


\section{Funding}

The present was co-supported by the Global High-tech Biomedicine Technology Development Program of the National Research Foundation \& Korea Health Industry Development Institute funded by the Korean government (grant nos. NRF-2 015M3D6A1065114 and NRF-2015M3D6A1065363).

\section{Availability of data and materials}

All data generated or analyzed during the present study are included in this published article.

\section{Authors' contributions}

DHB and EL designed the study. BCL, MJC, TRK, JHK, ESJ, WO, SKM and BCP performed the experiments and analyzed the data. JN and BJK contributed to drafting the manuscript. All authors have read and approved the manuscript and agree to be accountable for all aspects of the research in ensuring that the accuracy and integrity of any part of the work are appropriately investigated and resolved.

\section{Ethics approval and consent to participate}

All animal procedures were approved by the Institutional Animal Care and Use Committee of Chung-Ang University (approval no. 2018-00032) and conformed to all applicable National Institutes of Health guidelines. Neonatal hUCB-MSCs were collected from umbilical veins after neonatal delivery with informed consent of the pregnant mothers. This study was approved by the Institutional Review Board of MEDIPOST Co., Ltd.

\section{Patient consent for publication}

Not applicable.

\section{Competing interests}

The authors declare that they have no competing interests.

\section{References}

1. Ding DC, Chang YH, Shyu WC and Lin SZ: Human umbilical cord mesenchymal stem cells: A new era for stem cell therapy. Cell Transplant 24: 339-347, 2015.

2. Oh W, Kim DS, Yang YS and Lee JK: Immunological properties of umbilical cord blood-derived mesenchymal stromal cells. Cell Immunol 251: 116-123, 2008.

3. Chen M, Xiang Z and Cai J: The anti-apoptotic and neuro-protective effects of human umbilical cord blood mesenchymal stem cells (hUCB-MSCs) on acute optic nerve injury is transient. Brain Res 1532: 63-75, 2013.

4. Kang SG, Jeun SS, Lim JY, Kim SM, Yang YS, Oh WI, Huh PW and Park CK: Cytotoxicity of human umbilical cord blood-derived mesenchymal stem cells against human malignant glioma cells. Child's Nerv Syst 24: 293-302, 2008.

5. Lim JY, Jeong CH, Jun JA, Kim SM, Ryu CH, Hou Y, Oh W, Chang JW and Jeun SS: Therapeutic effects of human umbilical cord blood-derived mesenchymal stem cells after intrathecal administration by lumbar puncture in a rat model of cerebral ischemia. Stem Cell Res Ther 2: 38, 2011.

6. Kim JY, Jeon HB, Yang YS, Oh W and Chang JW: Application of human umbilical cord blood-derived mesenchymal stem cells in disease models. World J Stem Cells 2: 34-38, 2010.
7. Chung JY, Song M, Ha CW, Kim JA, Lee CH and Park YB: Comparison of articular cartilage repair with different hydrogel-human umbilical cord blood-derived mesenchymal stem cell composites in a rat model. Stem Cell Res Ther 5: 39, 2014.

8. Hillmer AM, Hanneken S, Ritzmann S, Becker T, Freudenberg J, Brockschmidt FF, Flaquer A, Freudenberg-Hua Y, Jamra RA, Metzen C, et al: Genetic variation in the human androgen receptor gene is the major determinant of common early-onset androgenetic alopecia. Am J Hum Genet 77: 140-148, 2005.

9. Lindner G, Botchkarev VA, Botchkareva NV, Ling G, van der Veen $\mathrm{C}$ and Paus R: Analysis of apoptosis during hair follicle regression (catagen). Am J Pathol 151: 1601, 1997.

10. Courtois M, Loussouarn G, Hourseau C and Grollier JF: Hair cycle and alopecia. Skin Pharmacol 7: 84-89, 1994.

11. Silverman MN and Sternberg EM: Glucocorticoid regulation of inflammation and its functional correlates: From HPA axis to glucocorticoid receptor dysfunction. Ann N Y Acad Sci 1261: 55-63, 2012.

12. Pérez P: Glucocorticoid receptors, epidermal homeostasis and hair follicle differentiation. Dermatoendocrinol 3: 166-174, 2011.

13. Slominski A, Wortsman J, Tuckey RC and Paus R: Differential expression of HPA axis homolog in the skin. Mol Cell Endocrinol 265: 143-149, 2007.

14. Zhang X, Yu M, Yu W, Weinberg J, Shapiro J and McElwee KJ: Development of alopecia areata is associated with higher central and peripheral hypothalamic-pituitary-adrenal tone in the skin graft induced C3H/HeJ mouse model. J Invest Dermatol 129: $1527-1538,2009$.

15. Ito N, Ito T, Kromminga A, Bettermann A, Takigawa M, Kees F, Straub RH and Paus R: Human hair follicles display a functional equivalent of the hypothalamic-pituitary-adrenal axis and synthesize cortisol. FASEB J 19: 1332-1334, 2005.

16. Arck PC, Handjiski B, Hagen E, Joachim R, Klapp BF and Paus R: Indications for a 'brain-hair follicle axis (BHA)': Inhibition of keratinocyte proliferation and up-regulation of keratinocyte apoptosis in telogen hair follicles by stress and substance P. FASEB J 15: 2536-2538, 2001.

17. Albus U: Guide for the Care and Use of Laboratory Animals (8th edition). SAGE Publications Sage UK, London, 2012.

18. Kwon TR, Kim JH, Hong JY, Seok J, Kim JM, Bak DH, Choi MJ, Mun SK, Kim CW and Kim BJ: Irradiation with $310 \mathrm{~nm}$ and $340 \mathrm{~nm}$ ultraviolet light-emitting-diodes can improve atopic dermatitis-like skin lesions in $\mathrm{NC} / \mathrm{Nga}$ mice. Photochem Photobiol Sci 17: 1127-1135, 2018.

19. Crema A, Ledda M, Fioretti D, Lolli MG, Sanchez M, Carico E, Marchese R, Rinaldi M and Lisi A: Combination of cord blood-derived human hepatic progenitors and hepatogenic factors strongly improves recovery after acute liver injury in mice through modulation of the Wnt/ $\beta$-catenin signaling. J Tissue Eng Regen Med 13: 1031-1043, 2019.

20. Park S, Erdogan S, Hwang D, Hwang S, Han EH and Lim YH: Bee venom promotes hair growth in association with inhibiting 5 $\alpha$-reductase expression. Biol Pharm Bull 39: 1060-1068, 2016.

21. Müller-Röver S, Handjiski B, van der Veen C, Eichmüller S, Foitzik K, McKay IA, Stenn KS and Paus R: A comprehensive guide for the accurate classification of murine hair follicles in distinct hair cycle stages. J Invest Dermatol 117: 3-15, 2001.

22. Choi W, Kwon SJ, Jin HJ, Jeong SY, Choi SJ, Oh W, Yang YS, Jeon HB and Jeon ES: Optimization of culture conditions for rapid clinical-scale expansion of human umbilical cord blood-derived mesenchymal stem cells. Clin Transl Med 6: 38, 2017.

23. Livak KJ and Schmittgen TD: Analysis of relative gene expression data using real-time quantitative PCR and the 2(-Delta Delta C(T)) method. Methods 25: 402-408, 2001.

24. Bjørk $\varnothing y$ G, Lamark T, Brech A, Outzen H, Perander M, Overvatn A, Stenmark H and Johansen T: P62/SQSTM1 forms protein aggregates degraded by autophagy and has a protective effect on huntingtin-induced cell death. J Cell Biol 171: 603-614, 2005.

25. Lippai $\mathrm{M}$ and Lőw P: The role of the selective adaptor p62 and ubiquitin-like proteins in autophagy. BioMed Res Int 2014: 832704, 2014.

26. Lachgar S, Charveron M, Gall Y and Bonafe J: Minoxidil upregulates the expression of vascular endothelial growth factor in human hair dermal papilla cells. Br J Dermatol 138: 407-411, 1998.

27. Ito M, Yang Z, Andl T, Cui C, Kim N, Millar SE and Cotsarelis G: Wnt-dependent de novo hair follicle regeneration in adult mouse skin after wounding. Nature 447: 316-320, 2007.

28. Chen Y and Yu L: Autophagic lysosome reformation. Exp Cell Res 319: 142-146, 2013. 
29. Young TH, Lee CY, Chiu HC, Hsu CJ and Lin SJ: Self-assembly of dermal papilla cells into inductive spheroidal microtissues on poly (ethylene-co-vinyl alcohol) membranes for hair follicle regeneration. Biomaterials 29: 3521-3530, 2008.

30. Sennett R and Rendl M: Mesenchymal-epithelial interactions during hair follicle morphogenesis and cycling. Semi Cell Dev Biol 23: 917-927, 2012.

31. Yoo BY, Shin YH, Yoon HH, Seo YK, Song KY and Park JK Application of mesenchymal stem cells derived from bone marrow and umbilical cord in human hair multiplication. J Dermatolol Sci 60: 74-83, 2010.

32. Kim Y, Jin HJ, Heo J, Ju H, Lee HY, Kim S, Lee S, Lim J, Jeong SY, Kwon J, et al: Small hypoxia-primed mesenchymal stem cells attenuate graft-versus-host disease. Leukemia 32: $2672,2018$.

33. Shin H, Ryu HH, Kwon O, Park BS and Jo SJ: Clinical use of conditioned media of adipose tissue-derived stem cells in female pattern hair loss: A retrospective case series study. Int J Dermatol 54: 730-735, 2015.

34. Hocking AM and Gibran NS: Mesenchymal stem cells: Paracrine signaling and differentiation during cutaneous wound repair. Exp Cell Res 316: 2213-2219, 2010.

35. Lai RC, Arslan F, Lee MM, Sze NS, Choo A, Chen TS, Salto-Tellez M, Timmers L, Lee CN, El Oakley RM, et al: Exosome secreted by MSC reduces myocardial ischemia/reperfusion injury. Stem Cell Res 4: 214-222, 2010.

36. Paus R, Handjiski B, Czarnetzki BM and Eichmüller S: A murine model for inducing and manipulating hair follicle regression (catagen): Effects of dexamethasone and cyclosporin A. J Invest Dermatol 103: 143-147, 1994.

37. Kwack MH, Lee JH, Seo CH, Kim JC, Kim MK and Sung YK: Dickkopf-1 is involved in dexamethasone-mediated hair follicle regression. Exp Dermatol 26: 952-954, 2017.

38. Premanand A and Rajkumari BR: Androgen modulation of Wnt/ $\beta$-catenin signaling in androgenetic alopecia. Arch Dermatol Res 310: 1-9, 2018.

39. Kishimoto J, Burgeson RE and Morgan BA: Wnt signaling maintains the hair-inducing activity of the dermal papilla. Genes Dev 14: 1181-1185, 2000

40. Huelsken J, Vogel R, Erdmann B, Cotsarelis G and Birchmeier W: $\beta$-Catenin controls hair follicle morphogenesis and stem cell differentiation in the skin. Cell 105: 533-545, 2001.

41. Iida M, Ihara S and Matsuzaki T: Hair cycle-dependent changes of alkaline phosphatase activity in the mesenchyme and epithelium in mouse vibrissal follicles. Dev Growth Differ 49 185-195, 2007.

42. Zhou L, Xu M, Yang Y, Yang K, Wickett RR, Andl T, Millar SE and Zhang Y: Activation of $\beta$-catenin signaling in CD133-positive dermal papilla cells drives postnatal hair growth. Plos One 11: e0160425, 2016
43. He J, Zhou J, Yang W, Zhou Q, Liang X, Pang X, Li J, Pan F and Liang H: Dexamethasone affects cell growth/apoptosis/chemosensitivity of colon cancer via glucocorticoid receptor $\alpha / \mathrm{NF}-\kappa \mathrm{B}$. Oncotarget 8: 67670-67683, 2017.

44. Boix J, Bigas J, Sevilla LM, Iacobone M, Citton M, Torresan F, Caroccia B, Rossi GP and Pérez P: Primary aldosteronism patients show skin alterations and abnormal activation of glucocorticoid receptor in keratinocytes. Sci Rep 7: 15806, 2017.

45. Deckers J, Bougarne N, Mylka V, Desmet S, Luypaert A, Devos M, Tanghe G, Van Moorleghem J, Vanheerswynghels M, De Cauwer L, et al: Co-Activation of glucocorticoid receptor and peroxisome proliferator-activated receptor- $\gamma$ in murine skin prevents worsening of atopic march. J Invest Dermatol 138: 1360-1370, 2018

46. Gauthier A, Fisch A, Seuwen K, Baumgarten B, Ruffner H, Aebi A, Rausch M, Kiessling F, Bartneck M, Weiskirchen R, et al: Glucocorticoid-loaded liposomes induce a pro-resolution phenotype in human primary macrophages to support chronic wound healing. Biomaterials 178: 481-495, 2018.

47. Vogt CJ and Schmid-Schönbein GW: Microvascular endothelial cell death and rarefaction in the glucocorticoid-induced hypertensive rat. Microcirculation 8: 129-139, 2001.

48. Llambi F and Green DR: Apoptosis and oncogenesis: Give and take in the BCL-2 family. Curr Opin Genet Dev 21: 12-20, 2011.

49. Czabotar PE, Lessene G, Strasser A and Adams JM: Control of apoptosis by the BCL-2 protein family: Implications for physiology and therapy. Nat Rev Mol Cell Biol 15: 49, 2014.

50. Palikaras K, Lionaki E and Tavernarakis N: Balancing mitochondrial biogenesis and mitophagy to maintain energy metabolism homeostasis. Cell Death Differ 22: 1399-1401, 2015.

51. Crotzer VL and Blum JS: Autophagy and adaptive immunity. Immunology 131: 9-17, 2010.

52. Levine B and Deretic V: Unveiling the roles of autophagy in innate and adaptive immunity. Nat Rev Immunol 7: 767-777, 2007.

53. Parodi C, Hardman JA, Allavena G, Marotta R, Catelani T, Bertolini M, Paus R and Grimaldi B: Autophagy is essential for maintaining the growth of a human (mini-) organ: Evidence from scalp hair follicle organ culture. Plos Biol 16: e2002864, 2018.

54. Jiang P and Mizushima N: LC3-and p62-based biochemical methods for the analysis of autophagy progression in mammalian cells. Methods 75: 13-18, 2015.

55. Wurzer B, Zaffagnini G, Fracchiolla D, Turco E, Abert C, Romanov J and Martens S: Oligomerization of p62 allows for selection of ubiquitinated cargo and isolation membrane during selective autophagy. Elife 4: e08941, 2015.

This work is licensed under a Creative Commons Attribution-NonCommercial-NoDerivatives 4.0 International (CC BY-NC-ND 4.0) License. 